FACTA UNIVERSITATIS (NIŠ)

Ser. Math. Inform. Vol. 35, No 4 (2020), 1031-1047

https://doi.org/10.22190/FUMI2004031C

\title{
ANTI-INVARIANT RIEMANNIAN SUBMERSIONS FROM LOCALLY CONFORMAL KAEHLER MANIFOLDS *
}

\author{
Majid Ali Choudhary and Lamia Saeed Alqahtani
}

(C) 2020 by University of Niš, Serbia | Creative Commons Licence: CC BY-NC-ND

\begin{abstract}
Recently, Sahin [10] studied the anti-invariant Riemannian submersions from almost Hermitian manifolds onto Riemannian manifolds. In present work, these notions of anti-invariant and Lagrangian Riemannian submersions have been extended to locally conformal Kaehler manifolds. Certain decomposition results and the geometry of foliation have also been investigated.

Keywords: anti-invariant Riemannian submersions; almost Hermitian manifolds; Riemannian manifolds; Kaehler manifolds.
\end{abstract}

\section{Introduction}

Locally conformal Kaehler manifolds (shortly, l.c.K. manifolds) have been rich source of attraction for many years. Many geometers considered these manifolds and their submanifolds in different settings (for details see, [3] and [13]). On the other side, for any Riemannian manifold $\mathcal{M}$ and Riemannian manifold $\mathcal{B}$, the Riemannian submersion $\pi$ from $\mathcal{M}$ onto $\mathcal{B}$ was studied for very first time by B. O'Neil [6]. Gray [4], Ianus [5], Park ([7], [8]), Sahin ([11], [12]), Choudhary [2] etc. have also taken into consideration the geometry of Riemannian submersions for different structures on differentiable manifolds. Recently, anti-invariant Riemannian submersions have been taken into study from almost Hermitian manifolds onto Riemannian manifolds by B. Sahin [10].

In present work, these notions of anti-invariant and Lagrangian Riemannian submersions have been extended to locally conformal Kaehler manifolds. Certain decomposition results and the geometry of foliation have also been investigated.

Received November 04, 2019; accepted October 18, 2020

2020 Mathematics Subject Classification. Primary 53C15; Secondary 53B20, 53C43

*The first author was supported by DST, Govt. of India, through Inspire Fellowship No. DST/INSPIRE Fellowship/2009/[xxv]. 


\section{Preliminaries}

This section is preliminary in nature wherein we collect definitions and formulas that are to be used. We start with l.c.K. manifold.

Definition 2.1. [3] For Hermitian manifold $(\tilde{\mathcal{M}}, g)$ of dimension- $2 m$ and Kaehler 2 -form $\Omega$ holding for the relation

$$
\Omega(\mathcal{X}, \mathcal{Y})=g(\mathcal{X}, J \mathcal{Y})
$$

for all $\mathcal{X}, \mathcal{Y} \in \chi(\tilde{\mathcal{M}})$ and closed 1-form $\omega$ defined globally on manifold $\tilde{\mathcal{M}}$ such that

$$
d \Omega=\omega \wedge \Omega,
$$

the manifold $\tilde{\mathcal{M}}$ is known as locally conformal Kaehler manifold.

Here, $\omega$ is sign of the Lee form of $\tilde{\mathcal{M}}$. We have the following cases for $\omega$ :

- when $\omega$ is exact, $\tilde{\mathcal{M}}$ is globally conformal Kahler (g.c.K.) manifold,

- when $\omega=0, \tilde{\mathcal{M}}$ is Kaehler manifold.

One can observe that any l.c.K. manifold becomes g.c.K. manifold provided it is simply connected. Let us use $\sharp$ to represent the rising of the indices in association with the metric $g$, then for any l.c.K. manifold $\tilde{\mathcal{M}}, B_{1}=\omega^{\sharp}$ indicates the Lee vector field and satisfies

$$
g\left(\mathcal{X}, B_{1}\right)=\omega(\mathcal{X}) ; \forall \mathcal{X} \in \chi(\tilde{\mathcal{M}})
$$

[3] When we use $\theta=\omega o J$ for anti-Lee form and $A=-J B_{1}$ for anti-Lee vector field, respectively. Then

$$
\left(\tilde{\nabla}_{\mathcal{X}} J\right) \mathcal{Y}=\frac{1}{2}\left\{\theta(\mathcal{Y}) \mathcal{X}-\omega(\mathcal{Y}) J \mathcal{X}-g(\mathcal{X}, \mathcal{Y}) A-\Omega(\mathcal{X}, \mathcal{Y}) B_{1}\right\}
$$

$\forall \mathcal{X}, \mathcal{Y} \in \chi(\tilde{\mathcal{M}})$, where, $\tilde{\nabla}$ is used for the Levi Civita connection of $(\tilde{\mathcal{M}}, g)$.

Any map $\pi$ of $m$-dimensional Riemannian manifold $\left(\mathcal{M}^{m}, g\right)$ onto a $b^{\prime}$-dimensional Riemannian manifold $\left(\mathcal{B}^{b^{\prime}}, g_{\mathcal{B}}\right)$ with $m>b^{\prime}$ stands for a Riemannian submersion if $\pi$ has maximal rank and the lengths of horizontal vectors are preserved by differential $\pi_{*}$.

It is known that $\pi^{-1}\left(q^{\prime}\right), q^{\prime} \in \mathcal{B}$ is an $\left(m-b^{\prime}\right)$ dimensional submanifold of Riemannian manifold $\mathcal{M}$ and named as fibers. A vector field on $\mathcal{M}$ is said to be

- vertical provided it is always tangent to $\pi^{-1}\left(q^{\prime}\right)$;

- horizontal provided it is always orthogonal to $\pi^{-1}\left(q^{\prime}\right)$.

Next, we have 
Definition 2.2. [10] Let $\mathcal{X}$ represents a vector field on a Riemannian manifold $\mathcal{M}$, then $\mathcal{X}$ is known as basic if

- it is horizontal

- it is $\pi$-related to a vector field $\mathcal{X}_{*}$ on $\mathcal{B}$, that is, $\pi_{*} \mathcal{X}_{p_{1}}=\mathcal{X}_{* \pi\left(p_{1}\right)}, \forall p_{1} \in \mathcal{M}$.

Let us use $\mathcal{V}$ and $\mathcal{H}$ to denote the projection morphisms on $k e r \pi_{*}$ and $\left(k e r \pi_{*}\right)^{\perp}$, respectively. Then

Lemma 2.1. [6] When $\pi: \mathcal{M} \rightarrow \mathcal{B}$ represents a Riemannian submersion from a Riemannian manifold $\mathcal{M}$ onto a Riemannian manifold $\mathcal{B}$. Then

(a) $g(\mathcal{X}, \mathcal{Y})=g_{\mathcal{B}}\left(\mathcal{X}_{*}, \mathcal{Y}_{*}\right) o \pi$

(b) $\mathcal{H}[\mathcal{X}, \mathcal{Y}]$ of $[\mathcal{X}, \mathcal{Y}]$ is basic vector field corresponding to $\left[\mathcal{X}_{*}, \mathcal{Y}_{*}\right]$, i.e., $\left([\mathcal{X}, \mathcal{Y}]^{\mathcal{H}}\right)=$ $\left(\mathcal{X}_{*}, \mathcal{Y}_{*}\right)$

(c) when $V$ is vertical vector, $[V, \mathcal{X}]$ is also vertical,

(d) when $\nabla^{*}$ be the Levi-Civita connection on $\mathcal{B}, \mathcal{H}(\nabla \mathcal{X} \mathcal{Y})$ will be the basic vector field that corresponds to $\nabla_{\mathcal{X}_{*}^{*}}^{*} \mathcal{Y}_{*}$.

Here, $\mathcal{X}, \mathcal{Y}$ are considered as basic vector fields on $\mathcal{M}$.

[6] Let us denote by the symbols $\mathcal{T}$ and $\mathcal{A}$, O'Neills tensors for vector fields $E, F$ on $\mathcal{M}$ and by $\nabla$ the Levi-Civita connection of $g$ such that the following hold

$$
\begin{aligned}
& \mathcal{A}_{E} F=\mathcal{H} \nabla_{\mathcal{H} E} \mathcal{V} F+\mathcal{V} \nabla_{\mathcal{H} E} \mathcal{H} F \\
& \mathcal{T}_{E} F=\mathcal{H} \nabla_{\mathcal{V} E} \mathcal{V} F+\mathcal{V} \nabla_{\mathcal{V} E} \mathcal{H} F .
\end{aligned}
$$

The necessary and sufficient condition for Riemannian submersion $\pi: \mathcal{M} \rightarrow \mathcal{B}$ to be totally geodesic fibres is that $\mathcal{T}$ vanishes identically. Now, let us suppose that $\Gamma(T \mathcal{M})$ denotes the set of all sections on the tangent bundle $T \mathcal{M}$, then for any $E \in$ $\Gamma(T \mathcal{M}), \mathcal{T}_{E}$ and $\mathcal{A}_{E}$ represent skew-symmetric operators on $(\Gamma(T \mathcal{M}), g)$ reversing the horizontal and vertical distributions. One can observe that $\mathcal{T}$ is vertical, $\mathcal{T}_{E}=$ $\mathcal{T}_{\mathcal{V} E}$ and $\mathcal{A}$ is horizontal, $\mathcal{A}=\mathcal{A}_{\mathcal{H} E}$ and hold for the following ([6], [10])

$$
\begin{gathered}
\mathcal{T}_{\mathcal{U}} \mathcal{W}=\mathcal{T}_{\mathcal{W}} \mathcal{U}, \forall \mathcal{U}, \mathcal{W} \in \Gamma\left(k e r \pi_{*}\right) \\
\mathcal{A}_{\mathcal{X}} \mathcal{Y}=-\mathcal{A}_{\mathcal{Y}} \mathcal{X}=\frac{1}{2} \mathcal{V}[\mathcal{X}, \mathcal{Y}], \forall \mathcal{X}, \mathcal{Y} \in\left(\Gamma\left(k e r \pi_{*}\right)^{\perp}\right)
\end{gathered}
$$

Now we state the following lemma [10] 
Lemma 2.2. When $\mathcal{X}, \mathcal{Y} \in \Gamma\left(\left(k e r \pi_{*}\right)^{\perp}\right)$ and $\mathcal{W}, \mathcal{W}^{\prime} \in \Gamma\left(k e r \pi_{*}\right)$, we have the following relations:

(a) $\nabla_{\mathcal{W}} \mathcal{W}^{\prime}=\mathcal{T}_{\mathcal{W}} \mathcal{W}^{\prime}+\hat{\nabla}_{\mathcal{W}} \mathcal{W}^{\prime}$

(b) $\nabla_{\mathcal{W}} \mathcal{X}=\mathcal{H} \nabla_{\mathcal{W}} \mathcal{X}+\mathcal{T}_{\mathcal{W}} \mathcal{X}$

(c) $\nabla_{\mathcal{X}} \mathcal{W}=\mathcal{A}_{\mathcal{X}} \mathcal{W}+\mathcal{V} \nabla_{\mathcal{X}} \mathcal{W}$

(d) $\nabla_{\mathcal{X}} \mathcal{Y}=\mathcal{H} \nabla_{\mathcal{X}} \mathcal{Y}+\mathcal{A}_{\mathcal{X}} \mathcal{Y}$

where $\hat{\nabla}_{\mathcal{W}} \mathcal{W}^{\prime}=\mathcal{V} \nabla_{\mathcal{W}} \mathcal{W}^{\prime}$. Moreover, $\mathcal{H} \nabla_{\mathcal{W}} \mathcal{X}=\mathcal{A}_{\mathcal{X}} \mathcal{W}$, when $\mathcal{X}$ is basic.

\section{Anti-invariant and Lagrangian Riemannian submersions}

This section deals with the anti-invariant and Lagrangian Riemannian submersion. Certain conditions to show these submersions to be totally geodesic maps are also discussed. A diffeomorphism $f$ of Riemannian manifold $(\mathcal{M}, g)$ onto another Riemannian manifold $\left(\mathcal{B}, g^{\prime}\right)$ is said be geodesic map if image of any geodesic arc in $\mathcal{M}$ under $f$ is a geodesic arc in $\mathcal{B}$ and image of any geodesic arc in $\mathcal{B}$ under $f^{-1}$ is a geodesic arc in $\mathcal{M}$. A map is said to be totally geodesic if its hessian vanishes.

Now, recall anti-invariant Riemannian submersion by the following way.

Definition 3.1. $[10]$ Let $\left(\mathcal{M}, g_{\mathcal{M}}, J\right)$ represents a complex almost Hermitian manifold of dimension $m$ and $\left(\mathcal{B}, g_{\mathcal{B}}\right)$ be a Riemannian manifold. Then, any Riemannian submersion $\pi: \mathcal{M} \rightarrow \mathcal{B}$ is said to be anti-invariant Riemannian submersion if $J\left(k e r \pi_{*}\right) \subseteq\left(k e r \pi_{*}\right)^{\perp}$.

For an anti-invariant Riemannian submersion $\pi$ from an almost Hermitian manifold $\left(\mathcal{M}, g_{\mathcal{M}}, J\right)$ onto a Riemannian manifold $\left(\mathcal{B}, g_{\mathcal{B}}\right)$, above definition implies $J\left(k e r \pi_{*}\right)^{\perp} \cap\left(k e r \pi_{*}\right) \neq 0$, and that produces

$$
\left(k e r \pi_{*}\right)^{\perp}=J\left(k e r \pi_{*}\right) \oplus \mu,
$$

here $\mu$ is used for the orthogonal complementary distribution to $J\left(k e r \pi_{*}\right)$ in $\left(k e r \pi_{*}\right)^{\perp}$. So,

$$
J \mathcal{X}=B \mathcal{X}+C \mathcal{X}, \quad \mathcal{X} \in \Gamma\left(\left(k e r \pi_{*}\right)^{\perp}\right), B \mathcal{X} \in \Gamma\left(k e r \pi_{*}\right), C \mathcal{X} \in \Gamma(\mu)
$$

For Riemannian submersion $\pi,(3.2)$ and $\pi_{*}\left(\left(k e r \pi_{*}\right)^{\perp}\right)=T \mathcal{B}$ indicate

$$
g_{\mathcal{B}}\left(\pi_{*} J V, \pi_{*} C \mathcal{X}\right)=0, \quad \forall \mathcal{X} \in \Gamma\left(\left(k e r \pi_{*}\right)^{\perp}\right), \mathcal{W} \in \Gamma\left(k e r \pi_{*}\right),
$$

implying

$$
T \mathcal{B}=\pi_{*}\left(J\left(k e r \pi_{*}\right)\right) \oplus \pi_{*}(\mu) .
$$


[1] Let $\phi^{\prime}: \mathcal{M} \rightarrow \mathcal{B}$ be smooth map from Riemannian manifold $\left(\mathcal{M}, g_{\mathcal{M}}\right)$ onto $\left(\mathcal{B}, g_{\mathcal{B}}\right)$. Then, any section of the bundle $\operatorname{Hom}\left(T \mathcal{M}, \phi^{\prime-1}(T \mathcal{B})\right) \rightarrow \mathcal{M}$ can be thought by differential $\phi_{*}^{\prime}, \phi^{\prime-1}(T \mathcal{B})$ being the pullback bundle having fibres $\left(\phi^{\prime-1}(T B)\right)_{p}=$ $T_{\phi^{\prime}(p)} B, p \in \mathcal{M}$. Thanks to pullback connection and the Levi-Civita connection $\nabla^{\mathcal{M}}$, one can induce a connection $\nabla$ for $\operatorname{Hom}\left(T \mathcal{M}, \phi^{\prime-1}(T \mathcal{B})\right)$. Hence, define the second fundamental form of $\phi^{\prime}$ by

$$
\left(\nabla \phi_{*}^{\prime}\right)(\mathcal{X}, \mathcal{Y})=\nabla_{\mathcal{X}}^{\phi^{\prime}} \phi_{*}^{\prime}(\mathcal{Y})-\phi_{*}^{\prime}\left(\nabla_{\mathcal{X}}^{\mathcal{M}} \mathcal{Y}\right), \forall \mathcal{X}, \mathcal{Y} \in \Gamma(T \mathcal{M})
$$

here, $\Gamma(T \mathcal{M})$ represents set of all sections on the tangent bundle $T \mathcal{M}$ and $\nabla^{\phi^{\prime}}$ is the pullback connection.

Next, we give the following result.

Lemma 3.1. When $\pi: \mathcal{M} \rightarrow \mathcal{B}$ represents anti-invariant Riemannian submersion from l.c.K. manifold $(\mathcal{M}, g, J)$ to a Riemannian manifold $\left(\mathcal{B}, g_{\mathcal{B}}\right)$, and $\omega$ be closed 1 -form defined globally on $\mathcal{M}$, then for all $\mathcal{X}, \mathcal{Y} \in \Gamma\left(\left(k e r \pi_{*}\right)^{\perp}\right), \mathcal{W} \in \Gamma\left(k e r \pi_{*}\right)$, we have

(i) $g(C \mathcal{Y}, J \mathcal{W})=0$

(ii) $g\left(\nabla_{\mathcal{X}} C \mathcal{Y}, J \mathcal{W}\right)=-g\left(C \mathcal{Y}, J \mathcal{A}_{\mathcal{X}} \mathcal{W}\right)+\frac{1}{2} \omega(\mathcal{W}) g(C \mathcal{Y}, C \mathcal{X})$

(iii) $g\left(\nabla_{\mathcal{W}} B \mathcal{Y}, C \mathcal{X}\right)=g\left(C \mathcal{X}, \mathcal{T}_{\mathcal{W}} B \mathcal{Y}\right)=-g\left(B \mathcal{Y}, \mathcal{T}_{\mathcal{W}} C \mathcal{X}\right)$

Proof (i) Let $\mathcal{Y} \in \Gamma\left(\left(k e r \pi_{*}\right)^{\perp}\right)$ and $\mathcal{W} \in \Gamma\left(k e r \pi_{*}\right)$, then in the light of (3.2), we get

$$
\begin{aligned}
g(C \mathcal{Y}, J \mathcal{W}) & =g(J \mathcal{Y}-B \mathcal{Y}, J \mathcal{W}) \\
& =g(J \mathcal{Y}, J \mathcal{W})
\end{aligned}
$$

where the fact $B \mathcal{Y} \in \Gamma\left(k e r \pi_{*}\right)$ and $J \mathcal{W} \in \Gamma\left(\left(k e r \pi_{*}\right)^{\perp}\right)$ was used. Moreover, $g(J \mathcal{Y}, J \mathcal{W})=g(\mathcal{Y}, \mathcal{W})=0$ and this completes the proof.

(ii) Let us assume $B_{1} \in \Gamma\left(k e r \pi_{*}\right)$, then taking view of (2.1) and part (i), we get

$$
\begin{aligned}
g\left(\nabla_{\mathcal{X}} C \mathcal{Y}, J \mathcal{W}\right) & =-g\left(C \mathcal{Y}, \nabla_{\mathcal{X}} J \mathcal{W}\right) \\
& =-g\left(C \mathcal{Y}, J \nabla_{\mathcal{X}} \mathcal{W}\right)+\frac{1}{2} \omega(\mathcal{W}) g(C \mathcal{Y}, J \mathcal{X})
\end{aligned}
$$

$\forall \mathcal{X}, \mathcal{Y} \in \Gamma\left(\left(k e r \pi_{*}\right)^{\perp}\right), \mathcal{W} \in \Gamma\left(k e r \pi_{*}\right)$. Thanks to (3.2), we arrive

$$
\begin{aligned}
g\left(\nabla_{\mathcal{X}} C \mathcal{Y}, J \mathcal{W}\right) & =-g\left(C \mathcal{Y}, J \nabla_{\mathcal{X}} \mathcal{W}\right)+\frac{1}{2} \omega(\mathcal{W}) g(C \mathcal{Y}, B \mathcal{X}+C \mathcal{X}) \\
& =-g\left(C \mathcal{Y}, J \nabla_{\mathcal{X}} \mathcal{W}\right)+\frac{1}{2} \omega(\mathcal{W}) g(C \mathcal{Y}, C \mathcal{X})
\end{aligned}
$$

because $C \mathcal{Y} \in \Gamma(\mu)$ and $B \mathcal{X} \in \Gamma\left(k e r \pi_{*}\right)$. Taking use of Lemma 2.2 produces

$$
g\left(\nabla_{\mathcal{X}} C \mathcal{Y}, J \mathcal{W}\right)=-g\left(C \mathcal{Y}, J \mathcal{A}_{\mathcal{X}} \mathcal{W}\right)-\left(C \mathcal{Y}, J \mathcal{V} \nabla_{\mathcal{X}} \mathcal{W}\right)+\frac{1}{2} \omega(\mathcal{W}) g(C \mathcal{Y}, C \mathcal{X})
$$


that simplifies to

$$
g\left(\nabla_{\mathcal{X}} C \mathcal{Y}, J \mathcal{W}\right)=-g\left(C \mathcal{Y}, J \mathcal{A}_{\mathcal{X}} \mathcal{W}\right)+\frac{1}{2} \omega(\mathcal{W}) g(C \mathcal{Y}, C \mathcal{X})
$$

here, we used $J \mathcal{V} \nabla_{\mathcal{X}} \mathcal{W} \in \Gamma\left(J k e r \pi_{*}\right)$.

From here, we assume that $B_{1} \in\left(k e r \pi_{*}\right)$. We also assume horizontal vector fields to be basic whenever needed in the proofs. Now, let us move to study the integrability results of the horizontal distribution $\left(k e r \pi_{*}\right)^{\perp}$. Also, note that ker $\pi_{*}$ is integrable.

Theorem 3.1. When $\pi: \mathcal{M} \rightarrow \mathcal{B}$ represents anti-invariant Riemannian submersion from l.c.K. manifold $(\mathcal{M}, g, J)$ onto a Riemannian manifold $\left(\mathcal{B}, g_{\mathcal{B}}\right)$, then the following are equivalent:

(a) $\left(k e r \pi_{*}\right)^{\perp}$ is integrable

(b) $g_{\mathcal{B}}\left(\left(\nabla \pi_{*}\right)(\mathcal{Y}, B \mathcal{X}), \pi_{*} J \mathcal{W}\right)=g_{\mathcal{B}}\left(\left(\nabla \pi_{*}\right)(\mathcal{X}, B \mathcal{Y}), \pi_{*} J \mathcal{W}\right)+g\left(C \mathcal{Y}, J \mathcal{A}_{\mathcal{X}} \mathcal{W}\right)$

$$
\begin{aligned}
& -g\left(C \mathcal{X}, J \mathcal{A}_{\mathcal{Y}} \mathcal{W}\right)-\frac{1}{2} g\left(B \mathcal{Y}, B_{1}\right) g(\mathcal{X}, J \mathcal{W}) \\
& +\frac{1}{2} g\left(B \mathcal{X}, B_{1}\right) g(\mathcal{Y}, J \mathcal{W})
\end{aligned}
$$

(c) $g\left(\mathcal{A}_{\mathcal{Y}} B \mathcal{X}-\mathcal{A}_{\mathcal{X}} B \mathcal{Y}, J \mathcal{W}\right)=-g\left(C \mathcal{Y}, J \mathcal{A}_{\mathcal{X}} \mathcal{W}\right)+g\left(C \mathcal{X}, J \mathcal{A}_{\mathcal{Y}} \mathcal{W}\right)$

$$
+\frac{1}{2} g\left(B \mathcal{Y}, B_{1}\right) g(\mathcal{X}, J \mathcal{W})-\frac{1}{2} g\left(B \mathcal{X}, B_{1}\right) g(\mathcal{Y}, J \mathcal{W})
$$

$\forall \mathcal{X}, \mathcal{Y} \in \Gamma\left(\left(k e r \pi_{*}\right)^{\perp}\right), \mathcal{W} \in \Gamma\left(k e r \pi_{*}\right)$.

Proof. Taking account of definition 3.1, we see $J \mathcal{Y} \in \Gamma\left(k e r \pi_{*} \oplus \mu\right)$ and $J \mathcal{W} \in$ $\Gamma\left(\left(k e r \pi_{*}\right)^{\perp}\right)$ and hence with the help of $(2.1)$ for $\mathcal{X} \in \Gamma\left(\left(k e r \pi_{*}\right)^{\perp}\right)$, we reach at

$$
\begin{aligned}
g([\mathcal{X}, \mathcal{Y}], \mathcal{W})= & g(J[\mathcal{X}, \mathcal{Y}], J \mathcal{W}) \\
= & g\left(J \nabla_{\mathcal{X}} \mathcal{Y}, J \mathcal{W}\right)-g\left(J \nabla_{\mathcal{Y}} \mathcal{X}, J \mathcal{W}\right) \\
= & g\left(\nabla_{\mathcal{X}} J \mathcal{Y}, J \mathcal{W}\right)-\frac{1}{2} \theta(\mathcal{Y}) g(\mathcal{X}, J \mathcal{W}) \\
& -g\left(\nabla_{\mathcal{Y}} J \mathcal{X}, J \mathcal{W}\right)+\frac{1}{2} \theta(\mathcal{X}) g(\mathcal{Y}, J \mathcal{W}),
\end{aligned}
$$

$\forall \mathcal{Y} \in \Gamma\left(\left(k e r \pi_{*}\right)^{\perp}\right), \mathcal{W} \in \Gamma\left(k e r \pi_{*}\right)$. Here $\theta=\omega o J, \Omega(\mathcal{X}, \mathcal{Y})=g(\mathcal{X}, J \mathcal{Y})$ and $g\left(\mathcal{X}, B_{1}\right)=\omega(\mathcal{X})$, then $B_{1} \in \Gamma\left(k e r \pi_{*}\right)$ and $(3.2)$ produce

$$
\begin{aligned}
g([\mathcal{X}, \mathcal{Y}], \mathcal{W})= & g\left(\nabla_{\mathcal{X}} J \mathcal{Y}, J \mathcal{W}\right)-g\left(\nabla_{\mathcal{Y}} J \mathcal{X}, J \mathcal{W}\right)-\frac{1}{2} g\left(B \mathcal{Y}, B_{1}\right) g(\mathcal{X}, J \mathcal{W}) \\
& +\frac{1}{2} g\left(B \mathcal{X}, B_{1}\right) g(\mathcal{Y}, J \mathcal{W}) \\
= & g\left(\nabla_{\mathcal{X}} B \mathcal{Y}, J \mathcal{W}\right)+g\left(\nabla_{\mathcal{X}} C \mathcal{Y}, J \mathcal{W}\right)-g\left(\nabla_{\mathcal{Y}} B \mathcal{X}, J \mathcal{W}\right) \\
& -g\left(\nabla_{\mathcal{Y}} C \mathcal{X}, J \mathcal{W}\right)-\frac{1}{2} g\left(B \mathcal{Y}, B_{1}\right) g(\mathcal{X}, J \mathcal{W})+\frac{1}{2} g\left(B \mathcal{X}, B_{1}\right) g(\mathcal{Y}, J \mathcal{W}) .
\end{aligned}
$$


Because $\pi$ represents a Riemannian submersion, we conclude

$$
\begin{aligned}
g([\mathcal{X}, \mathcal{Y}], \mathcal{W})= & g\left(\pi_{*} \nabla_{\mathcal{X}} B \mathcal{Y}, \pi_{*} J \mathcal{W}\right)+g\left(\nabla_{\mathcal{X}} C \mathcal{Y}, J \mathcal{W}\right)-g_{\mathcal{B}}\left(\pi_{*} \nabla_{\mathcal{Y}} B \mathcal{X}, \pi_{*} J \mathcal{W}\right) \\
& -g\left(\nabla_{\mathcal{Y}} C \mathcal{X}, J \mathcal{W}\right)-\frac{1}{2} g\left(B \mathcal{Y}, B_{1}\right) g(\mathcal{X}, J \mathcal{W})+\frac{1}{2} g\left(B \mathcal{X}, B_{1}\right) g(\mathcal{Y}, J \mathcal{W})
\end{aligned}
$$

Taking into account Lemma 3.1, we arrive at

$$
\begin{aligned}
g([\mathcal{X}, \mathcal{Y}], \mathcal{W})= & g_{\mathcal{B}}\left(-\left(\nabla \pi_{*}\right)(\mathcal{X}, B \mathcal{Y})+\left(\nabla \pi_{*}\right)(\mathcal{Y}, B \mathcal{X}), \pi_{*} J \mathcal{W}\right) \\
& -g\left(C \mathcal{Y}, J \mathcal{A}_{\mathcal{X}} \mathcal{W}\right)+g\left(C \mathcal{X}, J \mathcal{A}_{\mathcal{Y}} \mathcal{W}\right) \\
& -\frac{1}{2} g\left(B \mathcal{Y}, B_{1}\right) g(\mathcal{X}, J \mathcal{W})+\frac{1}{2} g\left(B \mathcal{X}, B_{1}\right) g(\mathcal{Y}, J \mathcal{W})
\end{aligned}
$$

proving $(\mathrm{a}) \Leftrightarrow(\mathrm{b})$.

Next, taking into consideration Lemma 2.2, we derive

$$
\begin{aligned}
\left(\nabla \pi_{*}\right)(\mathcal{X}, B \mathcal{Y}) & -\left(\nabla \pi_{*}\right)(\mathcal{Y}, B \mathcal{X}) \\
& =-\pi_{*}\left(\nabla_{\mathcal{X}} B \mathcal{Y}\right)+\pi_{*}\left(\nabla_{\mathcal{Y}} B \mathcal{X}\right) \\
& =-\pi_{*}\left(\nabla_{\mathcal{X}} B \mathcal{Y}-\nabla_{\mathcal{Y}} B \mathcal{X}\right) \\
& =\pi_{*}\left(\mathcal{A}_{\mathcal{Y}} B \mathcal{X}-\mathcal{A}_{\mathcal{X}} B \mathcal{Y}\right),
\end{aligned}
$$

$\forall \mathcal{X}, \mathcal{Y} \in \Gamma\left(\left(k e r \pi_{*}\right)^{\perp}\right), \mathcal{W} \in \Gamma\left(k e r \pi_{*}\right)$. Simplification reduces to

$$
\begin{aligned}
g_{\mathcal{B}}\left(\left(\nabla \pi_{*}\right)(\mathcal{X}, B \mathcal{Y})\right. & \left.-\left(\nabla \pi_{*}\right)(\mathcal{Y}, B \mathcal{X}), \pi_{*} J \mathcal{W}\right) \\
& =g_{\mathcal{B}}\left(\pi_{*}\left(\mathcal{A}_{\mathcal{Y}} B \mathcal{X}-\mathcal{A}_{\mathcal{X}} B \mathcal{Y}\right), \pi_{*} J \mathcal{W}\right) \\
& =g\left(\mathcal{A}_{\mathcal{Y}} B \mathcal{X}-\mathcal{A}_{\mathcal{X}} B \mathcal{Y}, J \mathcal{W}\right)
\end{aligned}
$$

moreover, $\mathcal{A}_{\mathcal{X}} B \mathcal{Y}-\mathcal{A}_{\mathcal{Y}} B \mathcal{X} \in \Gamma\left(\left(k e r \pi_{*}\right)^{\perp}\right)$, it establishes $(\mathrm{b}) \Leftrightarrow(\mathrm{c})$.

Definition 3.2. [10] Let $\pi$ represents an anti-invariant Riemannian submersion such that $J\left(k e r \pi_{*}\right)=\left(k e r \pi_{*}\right)^{\perp}$. Then, $\pi$ is known as Lagrangian Riemannian submersion. Moreover, when $\mu \neq\{0\}, \pi$ is called as proper anti-invariant Riemannian submersion.

Thanks to Theorem 3.1, we write the following.

Corollary 3.1. When $\pi: \mathcal{M} \rightarrow \mathcal{B}$ represents a Lagrangian Riemannian submersion from l.c.K. manifold $(\mathcal{M}, g, J)$ onto a Riemannian manifold $\left(\mathcal{B}, g_{\mathcal{B}}\right)$, then the following are equivalent:

(a) $\left(k e r \pi_{*}\right)^{\perp}$ is integrable

(b) $\left(\nabla \pi_{*}\right)(\mathcal{X}, J \mathcal{Y})=\left(\nabla \pi_{*}\right)(\mathcal{Y}, J \mathcal{X})-\frac{1}{2} g\left(B \mathcal{Y}, B_{1}\right) \mathcal{X}+\frac{1}{2} g\left(B \mathcal{X}, B_{1}\right) \mathcal{Y}$

(c) $\pi_{*}\left(\mathcal{A}_{\mathcal{X}} J \mathcal{Y}-\mathcal{A}_{\mathcal{Y}} J \mathcal{X}\right)=\frac{1}{2} g\left(B \mathcal{Y}, B_{1}\right) \mathcal{X}-\frac{1}{2} g\left(B \mathcal{X}, B_{1}\right) \mathcal{Y}, \forall \mathcal{X}, \mathcal{Y} \in \Gamma\left(\left(k e r \pi_{*}\right)^{\perp}\right)$ 
Proof. Let us assume that $\mathcal{X}, \mathcal{Y} \in \Gamma\left(\left(k e r \pi_{*}\right)^{\perp}\right)$ and $\mathcal{W} \in \Gamma\left(k e r \pi_{*}\right)$. Then, $J \mathcal{X} \in \Gamma\left(k e r \pi_{*}\right)$ and $J \mathcal{W} \in \Gamma\left(\left(k e r \pi_{*}\right)^{\perp}\right)$. Hence, taking into light (2.1), we derive

$$
\begin{aligned}
g([\mathcal{X}, \mathcal{Y}], \mathcal{W})= & g(J[\mathcal{X}, \mathcal{Y}], J \mathcal{W}) \\
= & g\left(J \nabla_{\mathcal{X}} \mathcal{Y}, J \mathcal{W}\right)-g\left(J \nabla_{\mathcal{Y}} \mathcal{X}, J \mathcal{W}\right) \\
= & g\left(\nabla_{\mathcal{X}} J \mathcal{Y}, J \mathcal{W}\right)-g\left(\nabla_{\mathcal{Y}} J \mathcal{X}, J \mathcal{W}\right) \\
& -\frac{1}{2} g\left(B \mathcal{Y}, B_{1}\right) g(\mathcal{X}, J \mathcal{W})+\frac{1}{2} g\left(B \mathcal{X}, B_{1}\right) g(\mathcal{Y}, J \mathcal{W}) .
\end{aligned}
$$

Use of (3.4) produces

$$
\begin{aligned}
g([\mathcal{X}, \mathcal{Y}], \mathcal{W})= & g_{\mathcal{B}}\left(\pi_{*} \nabla_{\mathcal{X}} J \mathcal{Y}, \pi_{*} J \mathcal{W}\right)-g_{\mathcal{B}}\left(\pi_{*} \nabla_{\mathcal{Y}} J \mathcal{X}, \pi_{*} J \mathcal{W}\right) \\
& -\frac{1}{2} g\left(B \mathcal{Y}, B_{1}\right) g(\mathcal{X}, J \mathcal{W})+\frac{1}{2} g\left(B \mathcal{X}, B_{1}\right) g(\mathcal{Y}, J \mathcal{W}) \\
= & -g_{\mathcal{B}}\left(\left(\nabla \pi_{*}\right)(\mathcal{X}, J \mathcal{Y}), \pi_{*} J \mathcal{W}\right)+g_{\mathcal{B}}\left(\left(\nabla \pi_{*}\right)(\mathcal{Y}, J \mathcal{X}), \pi_{*} J \mathcal{W}\right) \\
& -\frac{1}{2} g\left(B \mathcal{Y}, B_{1}\right) g(\mathcal{X}, J \mathcal{W})+\frac{1}{2} g\left(B \mathcal{X}, B_{1}\right) g(\mathcal{Y}, J \mathcal{W})
\end{aligned}
$$

thus, $\left(k e r \pi_{*}\right)^{\perp}$ is integrable iff

$$
\begin{aligned}
g_{\mathcal{B}}\left(\left(\nabla \pi_{*}\right)(\mathcal{X}, J \mathcal{Y}), \pi_{*} J \mathcal{W}\right)= & g_{\mathcal{B}}\left(\left(\nabla \pi_{*}\right)(\mathcal{Y}, J \mathcal{X}), \pi_{*} J \mathcal{W}\right)-\frac{1}{2} g\left(B \mathcal{Y}, B_{1}\right) g(\mathcal{X}, J \mathcal{W}) \\
& +\frac{1}{2} g\left(B \mathcal{X}, B_{1}\right) g(\mathcal{Y}, J \mathcal{W})
\end{aligned}
$$

establishing $(\mathrm{a}) \Leftrightarrow(\mathrm{b})$.

Next, with the help of (3.4) we get

$$
\begin{aligned}
\left(\nabla \pi_{*}\right)(\mathcal{Y}, J \mathcal{X}) & -\left(\nabla \pi_{*}\right)(\mathcal{X}, J \mathcal{Y}) \\
& =-\pi_{*}\left(\nabla_{\mathcal{Y}} J \mathcal{X}\right)+\pi_{*}\left(\nabla_{\mathcal{X}} J \mathcal{Y}\right) \\
& =\pi_{*}\left(\mathcal{H}\left(\nabla_{\mathcal{X}} J \mathcal{Y}\right)-\mathcal{H}\left(\nabla_{\mathcal{Y}} J \mathcal{X}\right)\right) \\
& =\pi_{*}\left(\mathcal{A}_{\mathcal{X}} J \mathcal{Y}-\mathcal{A}_{\mathcal{Y}} J \mathcal{X}\right)
\end{aligned}
$$

$\forall \mathcal{X}, \mathcal{Y} \in \Gamma\left(\left(k e r \pi_{*}\right)^{\perp}\right)$. This concludes $(\mathrm{b}) \Leftrightarrow(\mathrm{c})$.

\section{Geometry of leaves}

The geometry of leaves of $\left(k e r \pi_{*}\right)$ and $\left(k e r \pi_{*}\right)^{\perp}$ of anti-invariant and Lagrangian Riemannian submersions are studies here. We have

Theorem 4.1. When $\pi: \mathcal{M} \rightarrow \mathcal{B}$ represents an anti-invariant Riemannian submersion from l.c.K. manifold $(\mathcal{M}, g, J)$ onto a Riemannian manifold $\left(\mathcal{B}, g_{\mathcal{B}}\right)$, then the following are equivalent:

(a) totally geodesic foliation on $\mathcal{M}$ is defined by $\left(k e r \pi_{*}\right)^{\perp}$ 
Anti-invariant Riemannian Submersions from Locally Conformal Kaehler Manifolds 1039

(b) $g\left(\mathcal{A}_{\mathcal{X}} B \mathcal{Y}, J \mathcal{W}\right)=g\left(C \mathcal{Y}, J \mathcal{A}_{\mathcal{X}} \mathcal{W}\right)-\frac{1}{2} \omega(\mathcal{W}) g(C \mathcal{Y}, C \mathcal{X})$

$$
+\frac{1}{2} g\left(B \mathcal{Y}, B_{1}\right) g(\mathcal{X}, J \mathcal{W})+\frac{1}{2} g(\mathcal{X}, \mathcal{Y}) g\left(B_{1}, \mathcal{W}\right)
$$

(c) $g_{\mathcal{B}}\left(\left(\nabla \pi_{*}\right)(\mathcal{X}, J \mathcal{Y}), \pi_{*} J \mathcal{W}\right)=-g\left(C \mathcal{Y}, J \mathcal{A}_{\mathcal{X}} \mathcal{W}\right)+\frac{1}{2} \omega(\mathcal{W}) g(C \mathcal{Y}, C \mathcal{X})$

$$
-\frac{1}{2} g\left(B \mathcal{Y}, B_{1}\right) g(\mathcal{X}, J \mathcal{W})-\frac{1}{2} g(\mathcal{X}, \mathcal{Y}) g\left(B_{1}, \mathcal{W}\right)
$$

$\forall \mathcal{X}, \mathcal{Y} \in \Gamma\left(\left(k e r \pi_{*}\right)^{\perp}\right), \mathcal{W} \in \Gamma\left(k e r \pi_{*}\right)$.

Proof. Taking into account (2.1), (3.2), Lemma 2.2 and Lemma 3.1, we write the following

$$
\begin{aligned}
g\left(\nabla_{\mathcal{X}} \mathcal{Y}, \mathcal{W}\right)= & g\left(J \nabla_{\mathcal{X}} \mathcal{Y}, J \mathcal{W}\right) \\
= & g\left(\nabla_{\mathcal{X}} J \mathcal{Y}, J \mathcal{W}\right)-\frac{1}{2} g\left(B \mathcal{Y}, B_{1}\right) g(\mathcal{X}, J \mathcal{W})-\frac{1}{2} g(\mathcal{X}, \mathcal{Y}) g\left(B_{1}, \mathcal{W}\right) \\
= & g\left(\nabla_{\mathcal{X}} B \mathcal{Y}, J \mathcal{W}\right)+g\left(\nabla_{\mathcal{X}} C \mathcal{Y}, J \mathcal{W}\right)-\frac{1}{2} g\left(B \mathcal{Y}, B_{1}\right) g(\mathcal{X}, J \mathcal{W}) \\
& -\frac{1}{2} g(\mathcal{X}, \mathcal{Y}) g\left(B_{1}, \mathcal{W}\right) \\
= & g\left(\mathcal{A}_{\mathcal{X}} B \mathcal{Y}, J \mathcal{W}\right)-g\left(C \mathcal{Y}, J \mathcal{A}_{\mathcal{X}} \mathcal{W}\right)+\frac{1}{2} \omega(\mathcal{W}) g(C \mathcal{Y}, C \mathcal{X}) \\
& -\frac{1}{2} g\left(B \mathcal{Y}, B_{1}\right) g(\mathcal{X}, J \mathcal{W})-\frac{1}{2} g(\mathcal{X}, \mathcal{Y}) g\left(B_{1}, \mathcal{W}\right)
\end{aligned}
$$

$\forall \mathcal{X}, \mathcal{Y} \in \Gamma\left(\left(k e r \pi_{*}\right)^{\perp}\right), \mathcal{W} \in \Gamma\left(k e r \pi_{*}\right)$. In this way, a totally geodesic foliation on $\mathcal{M}$ is defined by $\left(k e r \pi_{*}\right)^{\perp}$ iff

$$
\begin{aligned}
g\left(\mathcal{A}_{\mathcal{X}} B \mathcal{Y}, J \mathcal{W}\right)= & g\left(C \mathcal{Y}, J \mathcal{A}_{\mathcal{X}} \mathcal{W}\right)-\frac{1}{2} \omega(\mathcal{W}) g(C \mathcal{Y}, C \mathcal{X}) \\
& +\frac{1}{2} g\left(B \mathcal{Y}, B_{1}\right) g(\mathcal{X}, J \mathcal{W})+\frac{1}{2} g(\mathcal{X}, \mathcal{Y}) g\left(B_{1}, \mathcal{W}\right)
\end{aligned}
$$

concluding (a) $\Leftrightarrow(\mathrm{b})$. Next, with the help of (3.4), we derive

$$
\begin{aligned}
g\left(\mathcal{A}_{\mathcal{X}} B \mathcal{Y}, J \mathcal{W}\right)= & g\left(\nabla_{\mathcal{X}} B \mathcal{Y}, J \mathcal{W}\right) \\
= & g\left(\nabla_{\mathcal{X}} J \mathcal{Y}, J \mathcal{W}\right)-g\left(\nabla_{\mathcal{X}} C \mathcal{Y}, J \mathcal{W}\right) \\
= & g_{\mathcal{B}}\left(\pi_{*} \nabla_{\mathcal{X}} J \mathcal{Y}, \pi_{*} J \mathcal{W}\right)-g\left(\nabla_{\mathcal{X}} C \mathcal{Y}, J \mathcal{W}\right) \\
= & -g_{\mathcal{B}}\left(\left(\nabla \pi_{*}\right)(\mathcal{X}, J \mathcal{Y}), \pi_{*} J \mathcal{W}\right)+g_{\mathcal{B}}\left(\nabla_{\mathcal{X}}^{\pi} \pi_{*}(J \mathcal{Y}), \pi_{*} J \mathcal{W}\right) \\
& -g\left(\nabla_{\mathcal{X}} C \mathcal{Y}, J \mathcal{W}\right) \\
= & -g_{\mathcal{B}}\left(\left(\nabla \pi_{*}\right)(\mathcal{X}, J \mathcal{Y}), \pi_{*} J \mathcal{W}\right)+g\left(\nabla_{\mathcal{X}} C \mathcal{Y}, J \mathcal{W}\right)-g\left(\nabla_{\mathcal{X}} C \mathcal{Y}, J \mathcal{W}\right) \\
= & -g_{\mathcal{B}}\left(\left(\nabla \pi_{*}\right)(\mathcal{X}, J \mathcal{Y}), \pi_{*} J \mathcal{W}\right)
\end{aligned}
$$

proving $(\mathrm{b}) \Leftrightarrow(\mathrm{c})$.

For Lagrangian Riemannian submersion, we have the following corollary.

Corollary 4.1. When $\pi$ denotes a Lagrangian Riemannian submersion from l.c.K. manifold $(\mathcal{M}, g, J)$ onto a Riemannian manifold $\left(\mathcal{B}, g_{\mathcal{B}}\right)$, then the following are equivalent: 
(a) totally geodesic foliation is defined by $\left(\text { ker } \pi_{*}\right)^{\perp}$ on manifold $\mathcal{M}$

(b) $g_{\mathcal{B}}\left(\mathcal{A}_{\mathcal{X}} J \mathcal{Y}, J \mathcal{W}\right)=\frac{1}{2} g\left(J \mathcal{Y}, B_{1}\right) g(\mathcal{X}, J \mathcal{W})+\frac{1}{2} g(\mathcal{X}, \mathcal{Y}) g\left(B_{1}, \mathcal{W}\right)$

(c) $g_{\mathcal{B}}\left(\left(\nabla \pi_{*}\right)(\mathcal{X}, J \mathcal{Y}), \pi_{*} J \mathcal{W}\right)=-\frac{1}{2} g\left(J \mathcal{Y}, B_{1}\right) g(\mathcal{X}, J \mathcal{W})-\frac{1}{2} g(\mathcal{X}, \mathcal{Y}) g\left(B_{1}, \mathcal{W}\right)$

$\forall \mathcal{X}, \mathcal{Y} \in \Gamma\left(\left(k e r \pi_{*}\right)^{\perp}\right), \mathcal{W} \in \Gamma\left(k e r \pi_{*}\right)$.

Proof. Thanks to (2.1), we write

$$
\begin{aligned}
g\left(\nabla_{\mathcal{X}} \mathcal{Y}, \mathcal{W}\right) & =g\left(J \nabla_{\mathcal{X}} \mathcal{Y}, J \mathcal{W}\right) \\
& =g\left(\nabla_{\mathcal{X}} J \mathcal{Y}, J \mathcal{W}\right)-\frac{1}{2} \theta(\mathcal{Y}) g(\mathcal{X}, J \mathcal{W})-\frac{1}{2} g(\mathcal{X}, \mathcal{Y}) g\left(B_{1}, \mathcal{W}\right) \\
& =g_{\mathcal{B}}\left(\pi_{*} \nabla_{\mathcal{X}} J \mathcal{Y}, \pi_{*} J \mathcal{W}\right)-\frac{1}{2} \theta(\mathcal{Y}) g(\mathcal{X}, J \mathcal{W})-\frac{1}{2} g(\mathcal{X}, \mathcal{Y}) g\left(B_{1}, \mathcal{W}\right) \\
& =g_{\mathcal{B}}\left(\pi_{*}\left(\mathcal{A}_{\mathcal{X}} J \mathcal{Y}\right), \pi_{*} J \mathcal{W}\right)-\frac{1}{2} \theta(\mathcal{Y}) g(\mathcal{X}, J \mathcal{W})-\frac{1}{2} g(\mathcal{X}, \mathcal{Y}) g\left(B_{1}, \mathcal{W}\right),
\end{aligned}
$$

$\forall \mathcal{X}, \mathcal{Y} \in \Gamma\left(\left(k e r \pi_{*}\right)^{\perp}\right), \mathcal{W} \in \Gamma\left(k e r \pi_{*}\right)$. This way, a totally geodesic foliation is defined by $\left(k e r \pi_{*}\right)^{\perp}$ on the manifold $\mathcal{M}$ iff

$$
g_{\mathcal{B}}\left(\mathcal{A}_{\mathcal{X}} J \mathcal{Y}, J \mathcal{W}\right)=\frac{1}{2} \theta(\mathcal{Y}) g(\mathcal{X}, J \mathcal{W})+\frac{1}{2} g(\mathcal{X}, \mathcal{Y}) g\left(B_{1}, \mathcal{W}\right) .
$$

Therefore, (a) $\Leftrightarrow(\mathrm{b})$. Next, taking help of (3.4) it follows

$$
\begin{aligned}
g_{\mathcal{B}}\left(\mathcal{A}_{\mathcal{X}} J \mathcal{Y}, J \mathcal{W}\right) & =g_{\mathcal{B}}\left(\nabla_{\mathcal{X}} J \mathcal{Y}, J \mathcal{W}\right) \\
& =g_{\mathcal{B}}\left(\pi_{*} \nabla_{\mathcal{X}} J \mathcal{Y}, \pi_{*} J \mathcal{W}\right) \\
& =-g_{\mathcal{B}}\left(\left(\nabla \pi_{*}\right)(\mathcal{X}, J \mathcal{Y}), \pi_{*} J \mathcal{W}\right)
\end{aligned}
$$

establishing

$$
g_{\mathcal{B}}\left(\left(\nabla \pi_{*}\right)(\mathcal{X}, J \mathcal{Y}), \pi_{*} J \mathcal{W}\right)=-\frac{1}{2} \theta(\mathcal{Y}) g(\mathcal{X}, J \mathcal{W})-\frac{1}{2} g(\mathcal{X}, \mathcal{Y}) g\left(B_{1}, \mathcal{W}\right)
$$

and that proves $(\mathrm{b}) \Leftrightarrow(\mathrm{c})$.

Now, taking into consideration (3.4) to get

$$
\left(\nabla \pi_{*}\right)(\mathcal{W}, \mathcal{X})=\nabla_{\mathcal{W}}^{\pi} \pi_{*} \mathcal{X}-\pi_{*} \nabla_{\mathcal{W}} \mathcal{X}, \quad \mathcal{X} \in \Gamma(\mu), \mathcal{W} \in \Gamma\left(k e r \pi_{*}\right) .
$$

Also,

$$
\left(\nabla \pi_{*}\right)(\mathcal{X}, \mathcal{W})=\nabla_{\mathcal{X}}^{\pi} \pi_{*} \mathcal{W}-\pi_{*} \nabla_{\mathcal{X}} \mathcal{W}
$$

We use above two equations and symmetric property of second fundamental form to get

$$
\nabla_{\mathcal{W}}^{\pi} \pi_{*} \mathcal{X}=0
$$

Next, we state the following Theorem. 
Theorem 4.2. When $\pi$ denotes an anti-invariant Riemannian submersion from l.c.K. manifold $(\mathcal{M}, g, J)$ onto a Riemannian manifold $\left(\mathcal{B}, g_{\mathcal{B}}\right)$, then the following are equivalent:

(a) totally geodesic foliation on $\mathcal{M}$ is defined by $\left(k e r \pi_{*}\right)$

(b) $\mathcal{T}_{\mathcal{W}} B \mathcal{X}+\mathcal{A}_{C} \mathcal{X} \mathcal{W}=0$ or $\mathcal{T}_{\mathcal{W}} B \mathcal{X}+\mathcal{A}_{C} \mathcal{X} \mathcal{W} \in \Gamma(\mu)$

(c) $g_{\mathcal{B}}\left(\left(\nabla \pi_{*}\right)(\mathcal{W}, J \mathcal{X}), \pi_{*} J \mathcal{W}^{\prime}\right)=0, \forall \mathcal{X} \in \Gamma\left(\left(k e r \pi_{*}\right)^{\perp}\right), \mathcal{W}, \mathcal{W}^{\prime} \in \Gamma\left(k e r \pi_{*}\right)$.

Proof. Taking into use (2.1), We obtain

$$
\begin{aligned}
g\left(\nabla_{\mathcal{W}} \mathcal{W}^{\prime}, \mathcal{X}\right) & =g\left(J \nabla_{\mathcal{W}} \mathcal{W}^{\prime}, J \mathcal{X}\right) \\
& =g\left(\nabla_{\mathcal{W}} J \mathcal{W}^{\prime}, J \mathcal{X}\right) \\
& =-g\left(J \mathcal{W}^{\prime}, \nabla_{\mathcal{W}} J \mathcal{X}\right), \quad \mathcal{X} \in \Gamma\left(\left(k e r \pi_{*}\right)^{\perp}\right), \mathcal{W}, \mathcal{W}^{\prime} \in \Gamma\left(k e r \pi_{*}\right)
\end{aligned}
$$

where orthogonality between $\left(k e r \pi_{*}\right)$ and $\left(k e r \pi_{*}\right)^{\perp}$ has been used. Taking help of (3.2) and Lemma 2.2, above equation reduces to

$$
\begin{aligned}
g\left(\nabla_{\mathcal{W}} \mathcal{W}^{\prime}, \mathcal{X}\right) & =-g\left(J \mathcal{W}^{\prime}, \nabla_{\mathcal{W}} B \mathcal{X}\right)-g\left(J \mathcal{W}^{\prime}, \nabla_{\mathcal{W}} C \mathcal{X}\right) \\
& =-g\left(J \mathcal{W}^{\prime}, \mathcal{T}_{\mathcal{W}} B \mathcal{X}\right)-g\left(J \mathcal{W}^{\prime}, \mathcal{A}_{C \mathcal{X}} \mathcal{W}\right) \\
& =-g\left(J \mathcal{W}^{\prime}, \mathcal{T}_{\mathcal{W}} B \mathcal{X}+\mathcal{A}_{C \mathcal{X}} \mathcal{W}\right)
\end{aligned}
$$

implying (a) $\Leftrightarrow(\mathrm{b})$. Furthermore, (3.4) produces

$$
\begin{aligned}
g\left(\mathcal{T}_{\mathcal{W}} B \mathcal{X}, J \mathcal{W}^{\prime}\right) & +g\left(\mathcal{A}_{C \mathcal{X}} \mathcal{W}, J \mathcal{W}^{\prime}\right) \\
& =g\left(\mathcal{H}\left(\nabla_{\mathcal{W}} B \mathcal{X}\right), J \mathcal{W}^{\prime}\right)+g\left(\mathcal{H}\left(\nabla_{\mathcal{W}} C \mathcal{X}\right), J \mathcal{W}^{\prime}\right) \\
& =g\left(\nabla_{\mathcal{W}} B \mathcal{X}, J \mathcal{W}^{\prime}\right)+g\left(\nabla_{\mathcal{W}} C \mathcal{X}, J \mathcal{W}^{\prime}\right) \\
& =g_{\mathcal{B}}\left(\pi_{*} \nabla_{\mathcal{W}} B \mathcal{X}, \pi_{*} J \mathcal{W}^{\prime}\right)+g_{\mathcal{B}}\left(\pi_{*} \nabla_{\mathcal{W}} C \mathcal{X}, \pi_{*} J \mathcal{W}^{\prime}\right) \\
& =-g_{\mathcal{B}}\left(\left(\nabla \pi_{*}\right)(\mathcal{W}, B \mathcal{X}), \pi_{*} J \mathcal{W}^{\prime}\right)-g_{\mathcal{B}}\left(\left(\nabla \pi_{*}\right)(\mathcal{W}, C \mathcal{X}), \pi_{*} J \mathcal{W}^{\prime}\right) \\
& +g_{\mathcal{B}}\left(\nabla_{\mathcal{W}}^{\pi} \pi_{*} C \mathcal{X}, \pi_{*} J \mathcal{W}^{\prime}\right) .
\end{aligned}
$$

Taking into consideration (4.1), we get

$$
\begin{aligned}
g\left(\mathcal{T}_{\mathcal{W}} B \mathcal{X}, J \mathcal{W}^{\prime}\right) & +g\left(\mathcal{A}_{C \mathcal{X}} \mathcal{W}, J \mathcal{W}^{\prime}\right) \\
& =-g_{\mathcal{B}}\left(\left(\nabla \pi_{*}\right)(\mathcal{W}, B \mathcal{X}), \pi_{*} J \mathcal{W}^{\prime}\right)-g_{\mathcal{B}}\left(\left(\nabla \pi_{*}\right)(\mathcal{W}, C \mathcal{X}), \pi_{*} J \mathcal{W}^{\prime}\right) \\
& =-g_{\mathcal{B}}\left(\left(\nabla \pi_{*}\right)(\mathcal{W}, J \mathcal{X}), \pi_{*} J \mathcal{W}^{\prime}\right)
\end{aligned}
$$

concluding $(\mathrm{b}) \Leftrightarrow(\mathrm{c})$.

Now, for a Lagrangian Riemannian submersion $\pi,(3.3)$ interprets $T \mathcal{B}=\pi_{*}\left(J\left(\right.\right.$ ker $\left.\left.\pi_{*}\right)\right)$.

Corollary 4.2. When $\pi$ represents a Lagrangian Riemannian submersion from l.c.K. manifold $(\mathcal{M}, g, J)$ onto a Riemannian manifold $\left(\mathcal{B}, g_{\mathcal{B}}\right)$, then the following are equivalent: 
(a) totally geodesic foliation on $\mathcal{M}$ is defined by $\left(k e r \pi_{*}\right)$

(b) $\mathcal{T}_{\mathcal{W}} J \mathcal{W}^{\prime}=0$

(c) $\left(\nabla \pi_{*}\right)(\mathcal{W}, J \mathcal{X})=0$

for $\mathcal{X} \in \Gamma\left(\left(k e r \pi_{*}\right)^{\perp}\right)$ and $\mathcal{W}, \mathcal{W}^{\prime} \in \Gamma\left(k e r \pi_{*}\right)$.

Proof. In the light of Theorem 4.2, $(a) \Leftrightarrow(b)$ is obvious. For the proof of $(b) \Leftrightarrow(c)$, consider that $\left(k e r \pi_{*}\right)$ and $\left(k e r \pi_{*}\right)^{\perp}$ are orthogonal, then we write

$$
\begin{aligned}
g\left(\nabla_{V} J \mathcal{W}, J \mathcal{X}\right) & =-g\left(J \mathcal{W}, \nabla_{V} J \mathcal{X}\right) \\
& =-g_{\mathcal{B}}\left(\pi_{*} J \mathcal{W}, \pi_{*} \nabla_{V} J \mathcal{X}\right) \\
& =g_{\mathcal{B}}\left(\pi_{*} J \mathcal{W},\left(\nabla \pi_{*}\right)(V, J \mathcal{X})\right) \\
g\left(\mathcal{T}_{V} J \mathcal{W}, J \mathcal{X}\right) & =g_{\mathcal{B}}\left(\pi_{*} J \mathcal{W},\left(\nabla \pi_{*}\right)(V, J \mathcal{X})\right),
\end{aligned}
$$

here, we have taken help of (3.4) and Lemma 2.2. Further, $\mathcal{T}_{V} J \mathcal{W} \in \Gamma\left(k e r \pi_{*}\right)$ that provides the required result $(b) \Leftrightarrow(c)$.

Definition 4.1. [1] For a differential map $\pi$ from a Riemannian manifold $\mathcal{M}$ onto a Riemannian manifold $\mathcal{B}$, if $\nabla \pi_{*}=0$ holds, then $\pi$ is said to be is called totally geodesic.

Next, we have

Theorem 4.3. When $\pi$ is used to denote a Lagrangian Riemannian submersion from l.c.K. manifold $(\mathcal{M}, g, J)$ onto a Riemannian manifold $\left(\mathcal{B}, g_{\mathcal{B}}\right)$. Then $\pi$ represents a totally geodesic map iff

$$
\mathcal{T}_{\mathcal{W}} J \mathcal{W}^{\prime}+\frac{1}{2} \omega\left(\mathcal{W}^{\prime}\right) J \mathcal{W}+\frac{1}{2} g\left(\mathcal{W}, \mathcal{W}^{\prime}\right) A=0
$$

and

$$
\mathcal{A}_{\mathcal{X}} J \mathcal{W}^{\prime}+\frac{1}{2} \omega\left(\mathcal{W}^{\prime}\right) J \mathcal{X}+\frac{1}{2} \Omega\left(\mathcal{X}, \mathcal{W}^{\prime}\right) B_{1}=0,
$$

$\forall \mathcal{X}, \mathcal{Y} \in \Gamma\left(\left(k e r \pi_{*}\right)^{\perp}\right), \mathcal{W}, \mathcal{W}^{\prime} \in \Gamma\left(k e r \pi_{*}\right)$.

Proof. The following holds for a Riemannian submersion $\pi$

$$
\left(\nabla \pi_{*}\right)(\mathcal{X}, \mathcal{Y})=0 \quad \forall \mathcal{X}, \mathcal{Y} \in \Gamma\left(\left(k e r \pi_{*}\right)^{\perp}\right)
$$

In the light of (2.1), (3.4) and (4.1), we derive

$$
\begin{aligned}
\left(\nabla \pi_{*}\right)\left(\mathcal{W}, \mathcal{W}^{\prime}\right) & =\nabla_{\mathcal{W}}^{\pi} \pi_{*}\left(\mathcal{W}^{\prime}\right)-\pi_{*}\left(\nabla_{\mathcal{W}} \mathcal{W}^{\prime}\right) \\
& =-\pi_{*}\left(\nabla_{\mathcal{W}} \mathcal{W}^{\prime}\right) \\
& =\pi_{*}\left(J\left(J \nabla_{\mathcal{W}} \mathcal{W}^{\prime}\right)\right) \\
& =\pi_{*}\left(J\left(\nabla_{\mathcal{W}} J \mathcal{W}^{\prime}+\frac{1}{2} \omega\left(\mathcal{W}^{\prime}\right) J \mathcal{W}+\frac{1}{2} g\left(\mathcal{W}, \mathcal{W}^{\prime}\right) A\right)\right) \\
& =\pi_{*}\left(J\left(\mathcal{T}_{\mathcal{W}} J \mathcal{W}^{\prime}+\frac{1}{2} \omega\left(\mathcal{W}^{\prime}\right) J \mathcal{W}+\frac{1}{2} g\left(\mathcal{W}, \mathcal{W}^{\prime}\right) A\right)\right),
\end{aligned}
$$


$\forall \mathcal{W}, \mathcal{W}^{\prime} \in\left(k e r \pi_{*}\right)$.

Further, use of (3.4) produces

$$
\begin{aligned}
\left(\nabla \pi_{*}\right)\left(\mathcal{X}, \mathcal{W}^{\prime}\right) & =\nabla_{\mathcal{X}}^{\pi} \pi_{*}\left(\mathcal{W}^{\prime}\right)-\pi_{*}\left(\nabla_{\mathcal{X}} \mathcal{W}^{\prime}\right) \\
& =-\pi_{*}\left(\nabla_{\mathcal{X}} \mathcal{W}^{\prime}\right) \\
& =\pi_{*}\left(J\left(J \nabla_{\mathcal{X}} \mathcal{W}^{\prime}\right)\right) \\
& =\pi_{*}\left(J\left(\nabla_{\mathcal{X}} J \mathcal{W}^{\prime}+\frac{1}{2} \omega\left(\mathcal{W}^{\prime}\right) J \mathcal{X}+\frac{1}{2} \Omega\left(\mathcal{X}, \mathcal{W}^{\prime}\right) B_{1}\right)\right) \\
& =\pi_{*}\left(J\left(\mathcal{A}_{\mathcal{X}} J \mathcal{W}^{\prime}+\frac{1}{2} \omega\left(\mathcal{W}^{\prime}\right) J \mathcal{X}+\frac{1}{2} \Omega\left(\mathcal{X}, \mathcal{W}^{\prime}\right) B_{1}\right)\right)
\end{aligned}
$$

$\forall \mathcal{X} \in \Gamma\left(\left(k e r \pi_{*}\right)^{\perp}\right), \mathcal{W}^{\prime} \in\left(k e r \pi_{*}\right)$. Hence, the result holds in view of (4.2),(4.3) and (4.4) and singularity of $J$.

\section{Decomposition theorems}

[14] Let us use $\mathcal{M}$ to represent a manifold whose dimension is $m$ and by $\left(\chi^{t}\right)$ a system of coordinate neighborhoods used to cover $\mathcal{M}$ in such a way that if $\left(\chi^{t}\right)$ and $\left(\chi^{t_{1}}\right)$ be any two coordinate neighborhoods, then in their intersection we obtain

$$
\chi^{a_{1}}=\chi^{a_{1}}\left(\chi^{a}\right), \chi^{x_{1}}=\chi^{x_{1}}\left(\chi^{x}\right),
$$

with

$$
\left|\delta_{a} \chi^{a_{1}}\right| \neq 0,\left|\delta_{x} \chi^{x_{1}}\right| \neq 0
$$

here all the indices $a, b, \ldots$ run over $1,2, \ldots, p$ and $x, y, z, \ldots$ over $p+1, \ldots, p+q=m$. This type of system of coordinate neighborhoods is known as separating coordinate system and if such a system of coordinate neighborhoods exists then it defines a locally product structure on the manifold $\mathcal{M}$. A manifold $\mathcal{M}$ equipped with a locally product structure is known as locally product manifold.

Next, we define

Definition 5.1. [9] When $N=\mathcal{M} \times \mathcal{B}$ is a manifold with Riemannian metric tensor $g$ and $\mathcal{D}_{\mathcal{M}}$ and $\mathcal{D}_{\mathcal{B}}$ be the canonical foliations intersecting perpendicularly everywhere. Then

(i) the necessary and sufficient condition for $g$ to represent the metric tensor of a warped product $\mathcal{M} \times_{f^{\prime}} \mathcal{B}$ is that $\mathcal{D}_{\mathcal{M}}$ and $\mathcal{D}_{\mathcal{B}}$ denote the totally geodesic and spherical foliations, respectively.

(ii) the necessary and sufficient condition for $g$ to be metric tensor of a twisted product $\mathcal{M} \times{ }_{f}, \mathcal{B}$ is that $\mathcal{D}_{\mathcal{M}}$ and $\mathcal{D}_{\mathcal{B}}$ represent the totally geodesic and totally umbilical foliations, respectively

(iii) the necessary and sufficient condition for $g$ to be metric tensor of a usual product of Riemannian manifolds is that $\mathcal{D}_{\mathcal{M}}$ and $\mathcal{D}_{\mathcal{B}}$ are totally geodesic foliations. 
Thanks to Theorems 4.1 and 4.2 , we have

Theorem 5.1. When $\pi$ is used to denote an anti-invariant Riemannian submersion from l.c.K. manifold $(\mathcal{M}, g, J)$ onto a Riemannian manifold $\left(\mathcal{B}, g_{\mathcal{B}}\right)$. Then the necessary and sufficient condition for $\mathcal{M}$ to be locally product manifold is that the following hold

$$
\begin{aligned}
g_{\mathcal{B}}\left(\left(\nabla \pi_{*}\right)(\mathcal{X}, J \mathcal{Y}), \pi_{*} J \mathcal{W}\right)= & -g\left(C \mathcal{Y}, J \mathcal{A}_{\mathcal{X}} \mathcal{W}\right)+\frac{1}{2} \omega(\mathcal{W}) g(C \mathcal{Y}, C \mathcal{X}) \\
& -\frac{1}{2} g\left(B \mathcal{Y}, B_{1}\right) g(\mathcal{X}, J \mathcal{W})-\frac{1}{2} g(\mathcal{X}, \mathcal{Y}) g\left(B_{1}, \mathcal{W}\right)
\end{aligned}
$$

and

$$
g_{\mathcal{B}}\left(\left(\nabla \pi_{*}\right)(\mathcal{W}, J \mathcal{X}), \pi_{*} J \mathcal{W}^{\prime}\right)=0
$$

$\forall \mathcal{X}, \mathcal{Y} \in \Gamma\left(\left(k e r \pi_{*}\right)^{\perp}\right), \mathcal{W}, \mathcal{W}^{\prime} \in \Gamma\left(k e r \pi_{*}\right)$

Thanks to Corollaries 4.1 and 4.2 , we have

Theorem 5.2. When $\pi$ is used to denote a Lagrangian Riemannian submersion from l.c.K. manifold $(\mathcal{M}, g, J)$ onto a Riemannian manifold $\left(\mathcal{B}, g_{\mathcal{B}}\right)$. Then the necessary and sufficient condition for $\mathcal{M}$ to be locally product manifold is that the following hold

$$
g_{\mathcal{B}}\left(\left(\nabla \pi_{*}\right)(\mathcal{X}, J \mathcal{Y}), \pi_{*} J \mathcal{W}\right)=-\frac{1}{2} g\left(J \mathcal{Y}, B_{1}\right) g(\mathcal{X}, J \mathcal{W})-\frac{1}{2} g(\mathcal{X}, \mathcal{Y}) g\left(B_{1}, \mathcal{W}\right)
$$

and

$$
\mathcal{T}_{\mathcal{W}} J \mathcal{W}^{\prime}=0
$$

$\forall \mathcal{X}, \mathcal{Y} \in \Gamma\left(\left(k e r \pi_{*}\right)^{\perp}\right), \mathcal{W}, \mathcal{W}^{\prime} \in \Gamma\left(k e r \pi_{*}\right)$.

For twisted product manifold, we get

Theorem 5.3. When $\pi$ represents a Lagrangian Riemannian submersion from l.c.K. manifold $(\mathcal{M}, g, J)$ onto a Riemannian manifold $\left(\mathcal{B}, g_{\mathcal{B}}\right)$. Then the necessary and sufficient condition for $\mathcal{M}$ to be locally twisted product manifold of the form $\mathcal{M}_{\left(k e r \pi_{*}\right)^{\perp}} \times_{f^{\prime}} \mathcal{M}_{\left(k e r \pi_{*}\right)}$ is that the following relations hold

$$
\mathcal{T}_{\mathcal{W}} J \mathcal{X}=-g\left(\mathcal{X}, \mathcal{T}_{\mathcal{W}} \mathcal{W}\right)\|\mathcal{W}\|^{-2} J \mathcal{W}
$$

and

$$
g_{\mathcal{B}}\left(\mathcal{A}_{\mathcal{X}} J \mathcal{Y}, J \mathcal{W}\right)=\frac{1}{2} g\left(J \mathcal{Y}, B_{1}\right) g(\mathcal{X}, J \mathcal{W})+\frac{1}{2} g(\mathcal{X}, \mathcal{Y}) g\left(B_{1}, \mathcal{W}\right)
$$

$\forall \mathcal{X}, \mathcal{Y} \in \Gamma\left(\left(k e r \pi_{*}\right)^{\perp}\right), \mathcal{W}, \mathcal{W}^{\prime} \in \Gamma\left(k e r \pi_{*}\right)$. Here, $\mathcal{M}_{\left(k e r \pi_{*}\right)^{\perp}} \times_{f^{\prime}} \mathcal{M}_{\left(k e r \pi_{*}\right)}$ denote the integral manifold of the distributions $\left(k e r \pi_{*}\right)^{\perp}$ and $\left(k e r \pi_{*}\right)$. 
Proof. With the help of (2.1) and Lemma 2.2, we write

$$
\begin{aligned}
g\left(\nabla_{\mathcal{W}} \mathcal{W}^{\prime}, \mathcal{X}\right) & =-g\left(\nabla_{\mathcal{W}} \mathcal{X}, \mathcal{W}^{\prime}\right) \\
& =-g\left(J \nabla_{\mathcal{W}} \mathcal{X}, J \mathcal{W}^{\prime}\right) \\
& =-g\left(\nabla_{\mathcal{W}} J \mathcal{X}, J \mathcal{W}^{\prime}\right) \\
& =-g\left(\mathcal{T}_{\mathcal{W}} J \mathcal{X}, J \mathcal{W}^{\prime}\right), \quad \forall \mathcal{X} \in \Gamma\left(\left(k e r \pi_{*}\right)^{\perp}\right), \mathcal{W}, \mathcal{W}^{\prime} \in \Gamma\left(k e r \pi_{*}\right)
\end{aligned}
$$

where orthogonality of $\left(k e r \pi_{*}\right)^{\perp}$ and $\left(k e r \pi_{*}\right)$ has been used. Hence, we conclude that for any function $\lambda$ on $\mathcal{M}$, the condition of totally umbilicity holds for $\left(k e r \pi_{*}\right)$ iff

$$
\mathcal{T}_{\mathcal{W}} J \mathcal{X}=-\mathcal{X}(\lambda) J \mathcal{W}
$$

Therefore, taking in use (2.1), we obtain

$$
\begin{aligned}
g(-\mathcal{X}(\lambda) J \mathcal{W}, J \mathcal{W}) & =g\left(\mathcal{T}_{\mathcal{W}} J \mathcal{X}, J \mathcal{W}\right) \\
-\mathcal{X}(\lambda)\|\mathcal{W}\|^{2} & =g\left(\mathcal{T}_{\mathcal{W}} J \mathcal{X}, J \mathcal{W}\right) \\
& =g\left(\nabla_{\mathcal{W}} J \mathcal{X}, J \mathcal{W}\right) \\
& =g\left(J \nabla_{\mathcal{W}} \mathcal{X}, J \mathcal{W}\right) \\
& =-g\left(\mathcal{X}, \mathcal{T}_{\mathcal{W}} \mathcal{W}\right) \\
\mathcal{X}(\lambda) & =g\left(\mathcal{X}, \mathcal{T}_{\mathcal{W}} \mathcal{W}\right)\|\mathcal{W}\|^{-2} .
\end{aligned}
$$

In this way, (5.1) and (5.2) produce

$$
\mathcal{T}_{\mathcal{W}} J \mathcal{X}=-g\left(\mathcal{X}, \mathcal{T}_{\mathcal{W}} \mathcal{W}\right)\|\mathcal{W}\|^{-2} J \mathcal{W}
$$

and that proves the result with the help of Corollary 4.1.

Next, we give a non existence result of a twisted product manifold $\mathcal{M}_{\left(k e r \pi_{*}\right)} \perp \times_{f^{\prime}}$ $\mathcal{M}_{\left(k e r \pi_{*}\right)}$.

Theorem 5.4. There does not exist Lagrangian Riemannian submersion $\pi$ from l.c.K. manifold $(\mathcal{M}, g, J)$ onto a Riemannian manifold $\left(\mathcal{B}, g_{\mathcal{B}}\right)$ such that $\mathcal{M}$ is a locally proper twisted product manifold $\mathcal{M}_{\left(k e r \pi_{*}\right) \perp} \times_{f^{\prime}} \mathcal{M}_{\left(k e r \pi_{*}\right)}$.

Proof. Let $\pi$ denotes a Lagrangian Riemannian submersion from l.c.K. manifold $\mathcal{M}$ onto a Riemannian manifold $\mathcal{B}$ and $\mathcal{M}$ be representing a locally twisted product $\mathcal{M}_{\left(k e r \pi_{*}\right)^{\perp}} \times_{f^{\prime}} \mathcal{M}_{\left(k e r \pi_{*}\right)}$. Then, due to definition 5.1, $\mathcal{M}_{\left(k e r \pi_{*}\right)}$ and $\mathcal{M}_{\left(k e r \pi_{*}\right)} \perp$ will be representing totally geodesic and totally umbilical foliations, respectively. When $h$ denotes the second fundamental form of $\mathcal{M}_{\left(k e r \pi_{*}\right)}$, we write

$$
g\left(\nabla_{\mathcal{X}} \mathcal{Y}, \mathcal{W}\right)=g(h(\mathcal{X}, \mathcal{Y}), \mathcal{W}), \quad \forall \mathcal{X}, \mathcal{Y} \in \Gamma\left(\left(k e r \pi_{*}\right)^{\perp}\right), \mathcal{W} \in \Gamma\left(k e r \pi_{*}\right)
$$

When $H$ is used for the mean curvature vector field of $\mathcal{M}_{\left(k e r \pi_{*}\right)^{\perp}}$, then we deduce

$$
g\left(\nabla_{\mathcal{X}} \mathcal{Y}, \mathcal{W}\right)=g(H, \mathcal{W}) g(\mathcal{X}, \mathcal{Y})
$$


Taking (2.1) and lemma 2.2 into consideration, we present

$$
\begin{aligned}
g\left(\nabla_{\mathcal{X}} \mathcal{Y}, \mathcal{W}\right) & =-g\left(\mathcal{Y}, \nabla_{\mathcal{X}} \mathcal{W}\right) \\
& =-g\left(J \mathcal{Y}, J \nabla_{\mathcal{X}} \mathcal{W}\right) \\
& =-g\left(J \mathcal{Y}, \mathcal{A}_{\mathcal{X}} J \mathcal{W}+\frac{1}{2} \omega(\mathcal{W}) J \mathcal{X}\right)
\end{aligned}
$$

here we used the orthogonal property between $\left(k e r \pi_{*}\right)^{\perp}$ and $\left(k e r \pi_{*}\right)$. Therefore, (5.3) and (5.4) generate the following

$$
\begin{aligned}
g(H, \mathcal{W}) g(\mathcal{X}, \mathcal{Y}) & =-g\left(J \mathcal{Y}, \mathcal{A}_{\mathcal{X}} J \mathcal{W}+\frac{1}{2} \omega(\mathcal{W}) J \mathcal{X}\right) \\
g(H, \mathcal{W}) g(J \mathcal{Y}, J \mathcal{X}) & =-g\left(J \mathcal{Y}, \mathcal{A}_{\mathcal{X}} J \mathcal{W}+\frac{1}{2} \omega(\mathcal{W}) J \mathcal{X}\right) \\
-g(H, \mathcal{W})\|\mathcal{X}\|^{2} & =g\left(\mathcal{A}_{\mathcal{X}} J \mathcal{W}+\frac{1}{2} \omega(\mathcal{W}) J \mathcal{X}, J \mathcal{X}\right) \\
& =g\left(\nabla_{\mathcal{X}} J \mathcal{W}+\frac{1}{2} \omega(\mathcal{W}) J \mathcal{X}, J \mathcal{X}\right) \\
& =g\left(J \nabla_{\mathcal{X}} \mathcal{W}, J \mathcal{X}\right) \\
& =-g\left(\mathcal{W}, \nabla_{\mathcal{X}} \mathcal{X}\right)
\end{aligned}
$$

Finally, we reach to

$$
g(H, \mathcal{W})\|\mathcal{X}\|^{2}=g\left(\mathcal{W}, \mathcal{A}_{\mathcal{X}} \mathcal{X}\right)
$$

So, use of $(2.5)$ shows $\mathcal{A}_{\mathcal{X}} \mathcal{X}=0$, that is $g(H, \mathcal{W})\|\mathcal{X}\|^{2}=0$. But, $H \in \Gamma\left(k e r \pi_{*}\right)$ with Riemannian metric $g$ supply $H=0$ and that that means $\left(k e r \pi_{*}\right)^{\perp}$ is totally geodesic. That proves $\mathcal{M}$ to be usual product of Riemannian manifolds.

\section{REF EREN CES}

1. P. BAIRD and J. C. WOOD: Harmonic morphisms between Riemannian manifolds, London Mathematical Society Monographs, 29, Oxford University Press, The clarendon Press, Oxford, 2003.

2. M. A. Choudhary, M. J. Matehkolaee and M. Jamali: On submersion of CRsubmanifolds of l.c.q.K. manifold, ISRN Geometry, 2012, doi:10.5402/2012/309145.

3. S. Dragomir and L. Ornea: Locally Conformal Kahler Geometry, Basel: Birkhauser, 1998.

4. A. GRAY: Pseudo-Riemannian almost product manifolds and submersions, J. Math. Mech. 16 (1967), 715-737.

5. S. IAnus, R. Mazzocco and G. E. Vilcu: Riemannian submersions from quaternionic manifolds, Acta Appl. Math. 104 (2008), no. 1, 83-89.

6. B. O'. NeILl: The fundamental equations of a submersion, Michigan Math. J. 13 (1996), 459-469. 
Anti-invariant Riemannian Submersions from Locally Conformal Kaehler Manifolds 1047

7. K. S. PARK: H-slant submersions, Bull. Korean Math. Soc. 49 (2012), no. 2, 329-338.

8. K. S. PARK: H-semi-invariant submersions, Taiwanese J. Math. 16 (2012), no. 5, 1865-1878.

9. R. Ponge and H. ReckzIEgel: Twisted products in pseudo-Riemannian geometry, Geom. Dedicata, 48 (1993), no. 1, 15-25.

10. B. SAHIN: Anti-invariant Riemannian submersions from almost Hermitian manifolds, Central European Journal of Mathematics, 8 (2010), no. 3, 437-447.

11. B. SAHin: Slant submersions from almost Hermitian manifolds, Bull. Math. Soc. Sci. Math. Roumanie Tome 54(102), (2011), no. 1, 93-105.

12. B. SAHIN: Semi-invariant Riemannian submersions from almost Hermitian manifolds, Taiwanese J. Math. 17 (2013), no. 2, 629-659.

13. I. Vaisman: On Locally Conformal Almost Kaehler Manifolds, Israel J. Math. 24 (1976), 338-351.

14. K. YANO and M. Kon: Structures on manifolds, Worlds Scientific, Singapore, 1984.

Majid Ali Choudhary

Department of Mathematics

School of Sciences

Maulana Azad National Urdu University

Hyderabad, India

majid_alichoudhary@yahoo.co.in

Lamia Saeed Alqahtani

Department of Mathematics

Faculty of Science

King Abdulaziz University

Jeddah 21589, Saudi Arabia

lalqahtani@kau.edu.sa 